\title{
Fueling Research Transparency: Computational Note- books and the Discussion Section
}

\author{
Sierdjan Koster ${ }^{1}$, Francisco Rowe $^{2}$ \\ ${ }^{1}$ University of Groningen, Groningen, The Netherlands \\ ${ }^{2}$ University of Liverpool, Liverpool, United Kingdom \\ Received: February 18 2020/Accepted: March 42020
}

The results of academic research, often publicly funded, should be easily available for a wide audience that includes fellow researchers, policy makers, journalists and anyone who takes an interest. On top of this, the research itself should be done in a transparent way so that results can be reproduced or perhaps falsified, as transparent research is good practice across all academic disciplines. Arguably, however, it is even more central in fields such as Regional Science with salient and tangible implications for local, regional, national and international socio-economic policies. The advent of internet and the growing capacity of computers has increased both the possibilities and the societal demand for transparent science.

Accommodating transparent research in Regional Science is a cornerstone of REGION. By its very nature as an online and open access journal, the contributions are made available to everyone. As it does not charge any submission fees, the journal is inclusive and invites contributions from across the globe. Also, in the longstanding and successful Resources section, REGION aims to unlock available datasets, visualization techniques and empirical approaches for a large audience. We are now proud to present two new types of publication that further accommodate transparent research in Regional Science: The Discussion section, and Computational Notebooks (see Figure 1).

The Discussion section aims to accommodate contributions that reflect on Regional Science as a field of science. Such reflections can pertain to fields of study within regional science, research agendas, but also to the organization of the field including the position of young scientists, gender representation and geographical inclusiveness of Regional Science. We invite contributions that put forward ideas for the further development of the field, but also retrospective accounts are very welcome. This issue has the first item in the Discussion section, which puts forward a research agenda on Transport research that emanates from the NECTAR network (Geurs, Macharis 2019).

Computational notebooks aim to augment the replicability, reproducibility and reach of research in Regional Science. Computational notebooks allow one to present analyses and integrate code, specialised software, dependencies, results and descriptive text into a single 'computational narrative' to be shared, read and executed by others. The interactive and narrative nature of computational notebooks provides unique opportunities for sharing computational research, enabling reproducibility in published scientific research. This is particularly important at a time of increased complexity of scientific studies. Computational notebooks are also valuable vehicles for teaching and demonstration of analytical tools. They can augment the impact of research beyond its primary academic objectives by extending original analysis and by reaching non-academic communities interested in Regional Science. The interactivity of notebooks can engage policy makers and the general public in ways that standard academic journal publications cannot. 


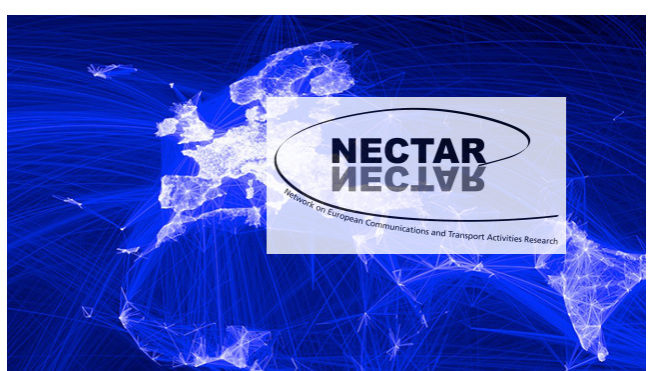

(a) Discussion Section

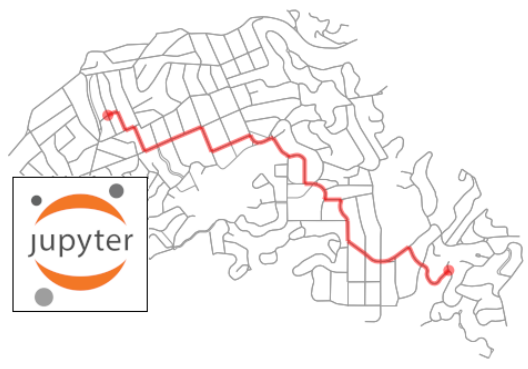

(b) Computable Notebooks

Figure 1: New publication types in REGION

REGION has recognised the potential of computational notebooks and the changing publication landscape, and we now facilitate the publication of computational notebooks, both as part of regular article submissions and as dedicated publications for the Resources section. We are proud to present the first contributions with computational notebooks in this issue (Comber 2020, Boeing 2020, Patias 2020) and we invite our contributors to incorporate computational notebooks in their submissions. In the near future, we will publish a dedicated special issue on the use of notebooks in the Regional Science.

We are confident that with these innovations, REGION can continue to play an active role in making Regional Science into a transparent and reproducible science.

\section{References}

Boeing G (2020) Urban street network analysis in a computational notebook. REGION 6[3]: 39-51. CrossRef.

Comber S (2020) Demonstrating the utility of machine learning innovations in address matching to spatial socio-economic applications. REGION 6[3]: 17-37. CrossRef.

Geurs K, Macharis C (2019) The future of european communication and transportation research: a research agenda. REGION 6[3]: D1-D21. CrossRef.

Patias N (2020) Exploring long-term youth unemployment in europe using sequence analysis: a reproducible notebook approach. REGION 6[3]: 53-69. CrossRef.

cc) (7) (5) (C) 2019 by the authors. Licensee: REGION - The Journal of ERSA, European Regional Science Association, Louvain-la-Neuve, Belgium. This article is distributed under the terms and conditions of the Creative Commons Attribution, Non-Commercial (CC BY NC) license (http://creativecommons.org/licenses/by-nc/4.0/). 